\title{
Single-Camera 3D PTV Methods for Evaporation-Driven Liquid Flows in Sessile Droplets
}

\section{Rossi, Massimiliano; Marin, Alvaro}

Published in:

Droplet Interactions and Spray Processes

Link to article, DOI:

10.1007/978-3-030-33338-6_18

Publication date:

2020

Document Version

Peer reviewed version

Link back to DTU Orbit

Citation (APA):

Rossi, M., \& Marin, A. (2020). Single-Camera 3D PTV Methods for Evaporation-Driven Liquid Flows in Sessile Droplets. In Droplet Interactions and Spray Processes (Vol. 121, pp. 225-236). Springer. Fluid Mechanics and Its Applications https://doi.org/10.1007/978-3-030-33338-6_18

\section{General rights}

Copyright and moral rights for the publications made accessible in the public portal are retained by the authors and/or other copyright owners and it is a condition of accessing publications that users recognise and abide by the legal requirements associated with these rights.

- Users may download and print one copy of any publication from the public portal for the purpose of private study or research.

- You may not further distribute the material or use it for any profit-making activity or commercial gain

- You may freely distribute the URL identifying the publication in the public portal 


\title{
Single-camera 3D PTV methods for evaporation-driven liquid flows in sessile droplets
}

\author{
Massimiliano Rossi ${ }^{\star 1}$, Alvaro Marin ${ }^{2}$ \\ ${ }^{1}$ Department of Physics, Technical University of Denmark, 2800 Kongens Lyngby, Denmark \\ 2Physics of Fluids Group, Max Planck Center for Complex Fluid Dynamics, University of \\ Twente, Enschede, The Netherlands \\ ${ }^{*}$ Corresponding author: rossi@fysik.dtu.dk
}

\begin{abstract}
The experimental characterization of the liquid flows in sessile evaporating droplets is an important task for the fundamental understanding of the complex phenomena occurring in these apparently simple systems. The liquid flow induced by the droplet evaporation has a strong three-dimensional character and conventional visualization methods are typically difficult to apply. A more effective approach is to look inside the droplets from the substrate where the droplet lies and use single-camera 3D particle tracking velocimetry (PTV) methods to reconstruct the whole flow field. This paper discusses the implementation of an experimental setup for the quantitative characterization of the flow inside sessile evaporating droplets based on two single-camera 3D PTV methods: the Astigmatic Particle Tracking Velocimetry (APTV) and the General Defocusing Particle Tracking (GDPT). Exemplary results on different types of sessile evaporating droplets are reported and discussed. The presented approach is easy to implement, does not require special or costly equipment, and has the potential to become a standard tool for this type of experiments.
\end{abstract}

\section{Introduction}

The evaporation of droplets of water or other liquids is a ubiquitous phenomenon in nature and in many engineering and technological processes. The internal and superficial flows that spontaneously develop during the evaporation process are not trivial and depend on the droplet composition, substrate material, temperature and humidity of the surrounding, and so on. The characterization and understanding of such flows is an active field of research and have important implications in many technological and biological processes, especially when the control or prediction of the shape and composition of the stain left at the end of the evaporation process is needed [1-3].

A classic example encountered in our everyday life is the ring-shaped stain observed when a spilled droplet of coffee dries out. Deegan et al. [4] in 1997 showed that particles are pushed to the ring-shaped stain due to a capillary flow that forces liquid (and the coffee particles dispersed in the liquid) toward the contact line to replenish the liquid lost during evaporation and maintain a minimal liquid-air surface (Fig. 1(a)). Additionally, the evaporation process cools down the liquid to a temperature lower than the one in substrate, therefore temperature and surface tension gradients build up across the droplet and drive Marangoni flow at the liquid-air surface [5]. In the case of water at room temperature, thermal Marangoni flow is directed from the contact line toward the drop summit and has a strong influence on the final particle deposit (Fig. 1(b)).

The presence of surfactants, mineral salts or surface-active components dissolved in the liquid phase also has a huge impact on the internal and superficial flows [6-7]. For example, 
chaotropic salts, such as $\mathrm{NaCl}$, when dissolve in polar liquids as water increase the surface tension. In an evaporating water salty droplet, salt quickly concentrates at the at the drop corner where the liquid evaporation rate is stronger. This can reverse the direction of the Marangoni flow and the circulation of the internal flow, which must comply with the volume conservation (Fig. 1(c)). Surfactants lead to even more complex and unpredictable scenarios, due to the complexity of surfactant adsorption/desorption from the interface, micelle formation in the bulk, and the continuous decrease of liquid/air interface during the evaporation process [8-9]. For this reason, very different liquid flow configurations can be observed depending on the nature of the surfactant and the stage of the droplet evaporation, as will be described below (Fig. 1(d)). Note that all cases discussed in the following chapters will use water as working liquid.

(a)

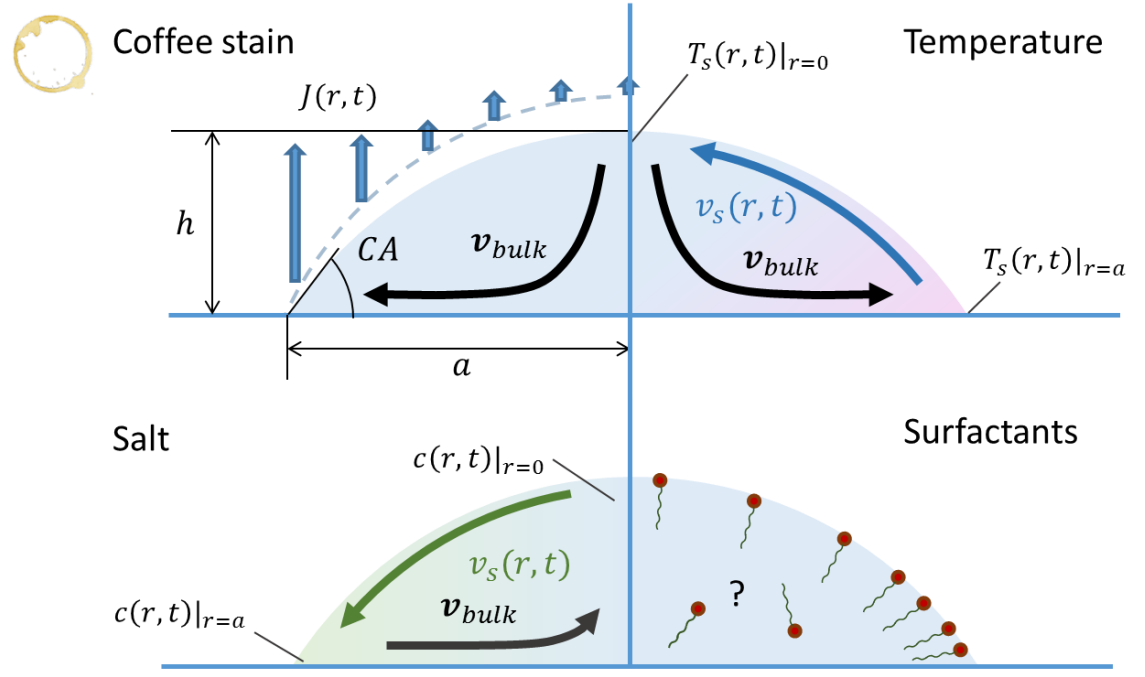

(b)

(d)

Fig. 1. Example of internal and superficial flows in sessile evaporating droplets. (a) Capillary flow responsible of the coffee-stain effect. (b) Marangoni flow driven by temperature gradients in the droplet. (c) Marangoni flow driven by concentration gradients of a chaotropic salt. (d) Surfactants alter the mobility of the water-air surface of the droplet in very complex way that yields different flow configurations depending on the surfactant nature and the stage of the droplet evaporation.

The experimental visualization or quantification of such flows is of paramount importance for the understanding of these phenomena and for the development and validation of numerical simulations or analytical models. Flow measurements inside evaporating droplets are, however, not an easy task, since conventional 2D or 3D visualization techniques fail when looking through a curved moving interface, as the drop interface. Another option is to look inside the drop from the bottom, but conventional methods will provide in this case a $2 \mathrm{D}$ projection of a complex 3D flow which is, in the best case scenario, difficult to interpret, but often gives a wrong picture of the flow field.

A solution is provided by the use of particle tracking methods based on a single-camera view, that allow to track the three-dimensional displacement of tracer particles dispersed in the fluid. In particular, methods based on defocusing have recently been used to characterize with unprecedented resolution the internal and superficial flow of different types of evaporating droplets [10-12]. In comparison with other methods such as confocal microscopy or holography, these methods do not need costly equipment and can be implemented using conventional inverted microscopes. Additionally, confocal microscopy and optical coherence tomography suffer of several constraints on both recording frame rates and total length of record, which can be easily avoided using single-camera 3D-PTV methods. In this work, we 
consider two methods, the Astigmatic Particle Tracking Velocimetry (APTV) and the General Defocusing Particle Tracking (GDPT), which have been successfully used to characterize the full flow field in evaporating droplets with excellent spatial and temporal resolution during the whole evaporation process. In Section 2, the general guidelines to implement a suitable experimental setup are discussed as well as the basic principles of the methods. In Section 3 , some examples of the results obtained with these methods on different types of sessile evaporating droplets are presented. Conclusions are provided in Section 4.

\section{Material and methods}

\subsection{Experimental setup}

A schematic of a general experimental setup to perform single-camera 3D PTV measurements on sessile evaporating droplets is depicted in Fig. 2. The droplets are gently deposited on a transparent substrate (typically a microscope slide) and then placed inside a measurement chamber. The first purpose of the chamber is to protect the droplet from unwanted air flows, the can significantly disturb the superficial and internal flows. For experiments where the active control of temperature or humidity is not required [11-12], their values can be approximated to the ones measured in the laboratory. In this case, it is important that the chamber is not sealed otherwise the relative humidity inside will increase due to the evaporation process, changing continuously the boundary conditions. If active control of temperature and humidity is required, the chamber must be equipped with a suitable feedback-control system. The temperature control can be achieved using Peltier elements or microscope incubators [10]. The equipment for full humidity control is more complicated and expensive, however, for chambers relatively small (less than 0.2 I with droplet diameters of $1-10 \mathrm{~mm}$ ), the vapour supply provided by the droplet is sufficient to rapidly saturate the chamber, therefore the humidity can be regulated simply by a controlled flow of dry air [10].

The global parameters to characterize the evaporation process, which are droplet radius, volume, and contact angle (CA), can be measured simultaneously from a side view of the droplet, as shown in Fig. 2. A teleobjective lens or a long-range microscope [10-12] are normally needed to achieve sufficient resolution. The drop radius, height, and CA can directly be measured after proper calibration from analysis of the side view images, whereas the volume can be derived from these parameters considering that sessile water droplets always assume a spherical-cap geometry, as long as the radius/height of the droplet is below the liquid's capillary length.

To perform the flow velocity measurement with particle tracking, the liquid droplets need to be previously seeded with tracer particles. The particles in the fluid are observed from the bottom through the transparent substrate by means of an inverted microscope. The optical system is chosen in order to have particle images with different shape depending on their depth position (as a consequence of defocusing or astigmatism, as described in the next section), therefore the full 3D displacement of each particle within a certain measurement volume can be determined. This approach allows to look at the internal flow of the droplets avoiding problems due to curved and moving surfaces, as it would be if the particles were observed from a top or side view. 


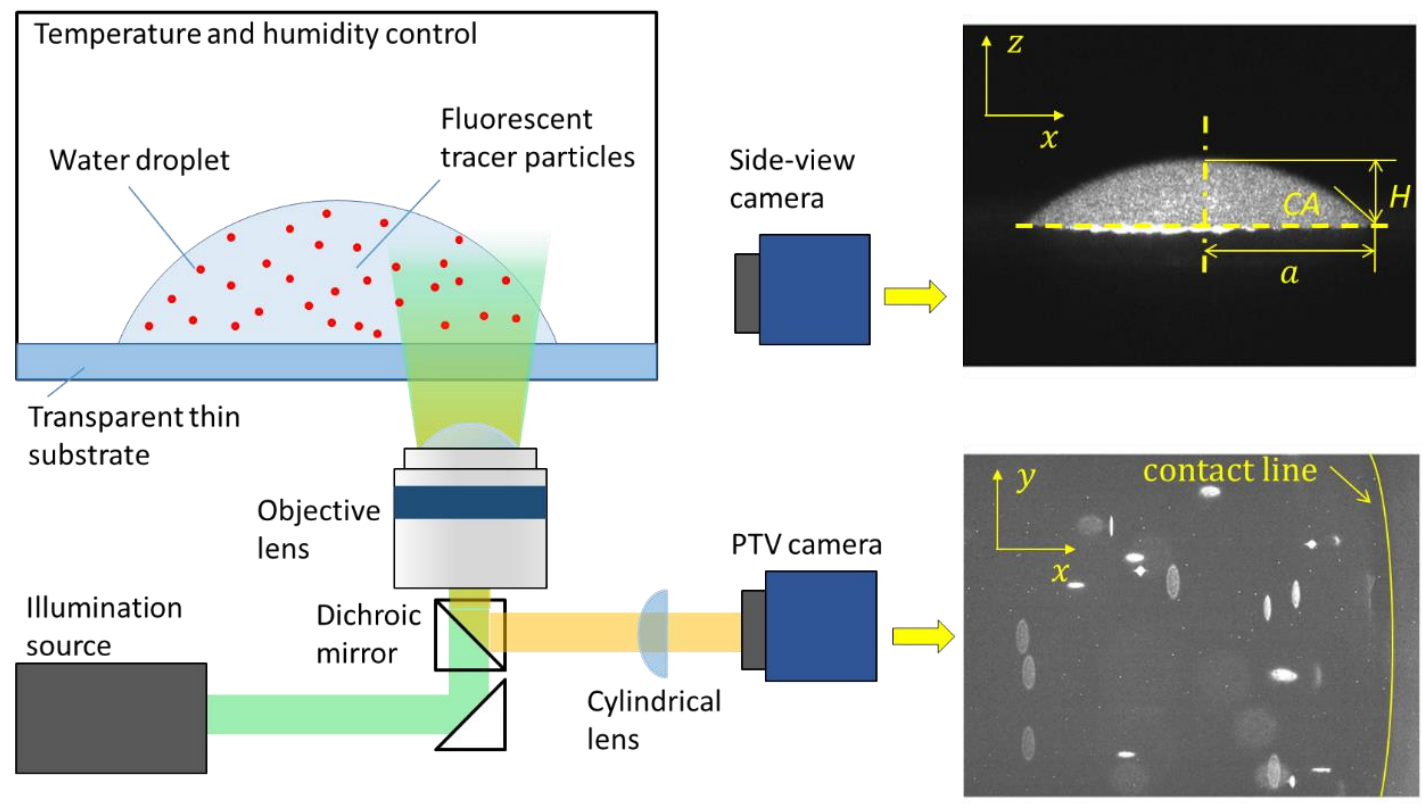

Fig. 2. Typical experimental setup. A liquid droplet is deposited on a transparent substrate placed inside a measurement chamber. The liquid is seeded with fluorescent tracer particles at small concentration, which are observed with an epifluorescent inverted microscope. The particles are at different depth positions therefore their images have different patterns depending on the degree of defocusing. This information is used to perform 3D particle tracking using a single-camera view [16]. Additionally, a cylindrical lens can be used to encode more efficiently the defocusing information [1315]. A side-view camera is used to measure the droplet profile and monitor the global drop parameters (volume, contact angle, radius) during the evaporation process.

As tracer particles, polystyrene spheres with a diameter of 1-2 $\mu \mathrm{m}$ functionalized with sulfate or carboxyl groups are typically used for measurements. The particle functionalization is specially important to disperse the polystyrene particles in polar liquids as water, which will be liquid used for the cases discussed. Smaller particles would be strongly affected by the Brownian motion, whereas larger particle will have a too large sedimentation velocity (the polystyrene density $\rho_{P S}=1050 \mathrm{~kg} / \mathrm{m} 3$ is slightly larger than the one of water). To improve the image quality, the particles are commonly labelled with a fluorescent dye and observed with an epifluorescent system. In such system, the particles are illuminated with a monochromatic light (provided by a laser or high-power LED) at the excitation wavelength of the fluorescent dye, and a suitable dichroic mirror with longpass filter is used to acquire images only by collecting the light emitted by the particles. With this approach, most of the noise due to reflection or background patterns can be eliminated, and clear bright particle images on a dark background can be obtained (see example figure in Fig. 2).

\subsection{Single-camera 3D PTV methods}

The single-camera 3D PTV methods used to obtain the results presented in the following section rely on the fact that the tracer particles in the droplet are observed with an optics with small depth of field (such as microscope objectives). In this case, the particle images are not in focus but have different degree of defocusing depending on their depth position. In general, the more a particle move away from the focal plane of the objective lens, the larger will be the diameter of the corresponding particle image. This information can be used to obtain the depth position of the particle. A simple measurement of the particle image diameter, however, is not 
sufficient to determine whether the particle is behind of after the focal plane, due to symmetry reasons. Therefore, more sophisticated approaches are needed.

(a)

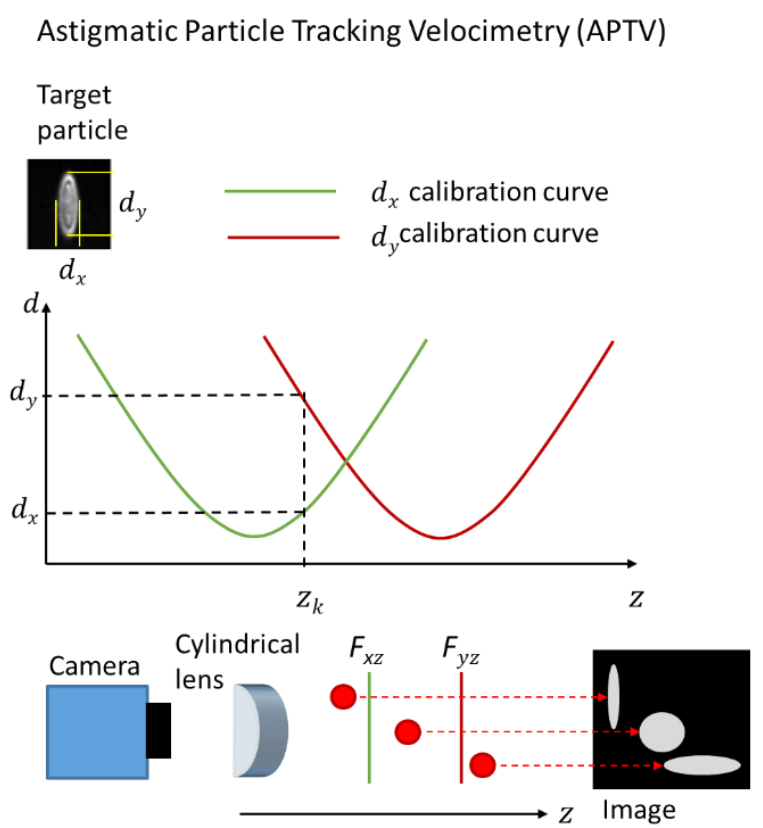

(b)

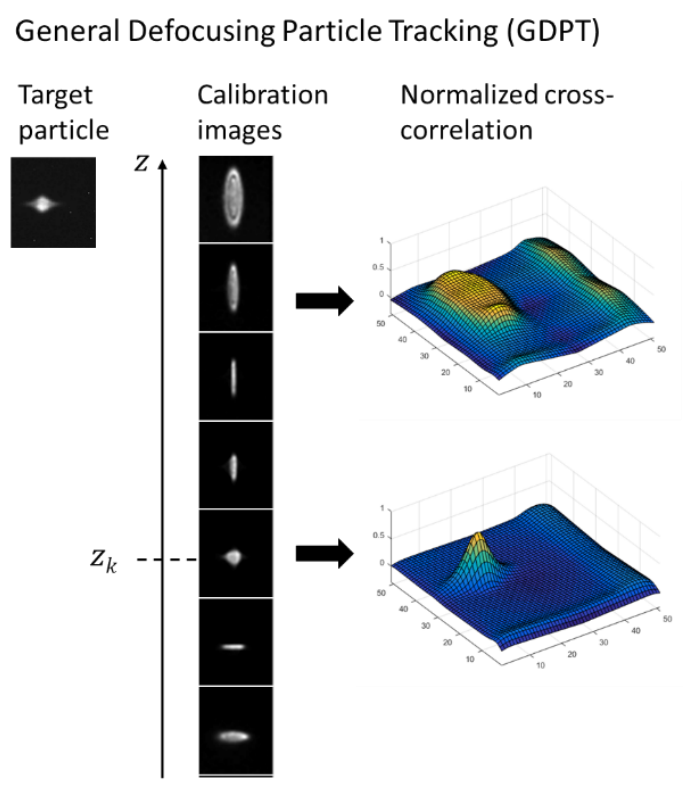

Fig. 3. Measurement methods for 3D particle tracking with a single camera. (a) Astigmatic Particle Tracking Velocimetry (APTV) [5-7]. Particle images obtained with astigmatic optics show characteristic elliptical shapes due to the fact the focal position in the horizontal direction is different from the one for the vertical direction. The depth position is derived from the measurement of the length of the major and minor axis of the elliptical particle images. (b) General Defocusing Particle Tracking (GDPT) $[4,9]$. This method is based on a look-up table that matches the particle image shapes with their respective depth positions. To identify the depth position of a target particle image, this is compared against the images in the look-up table using the normalized cross-correlation function, that rate the similarity between two images, with values from 0 to 1 (being 1 the perfect match).

Astigmatic Particle Image Velocimetry (APTV). A clever approach to encode more efficiently the defocusing information is to induce an astigmatic aberration in the optical path using a cylindrical lens in front of the camera sensor [13]. In this way, it does not exist a single focal plane anymore and a particle image will be in focus in the horizontal direction in a different position than in the vertical direction, resulting in elliptical particle images. From the measurement of the length of major and minor axis of the particle images is then possible to measure the corresponding depth position of the particle (see Fig.2 and Refs. [14-15]).

General Defocusing Particle Tracking (GDPT). A more general approach is to create a lookup table that maps the different particle images with their depth positions. Afterwards, a pattern recognition algorithm can be used to compare the recorded particle image with the ones in the look-up table and obtain the depth position (see Fig.2 and Ref [16]). This approach has the advantage to be completely general and to rely only on the assumption that the particle images change in a systematic fashion with respect to their depth position. GDPT can work well with astigmatic optics but also with conventional optics that show a certain degree of spherical aberration (present in most conventional lenses). The spherical aberration introduces also an asymmetry in the particle image shape depending if they are behind or after the focal plane 
[17]. More information on this method and a freely-available software for GDPT analysis can be found in [18].

\section{Results and discussion}

The results presented in this section have been obtained using the general experimental setup described in Section 2, and GDPT or APTV as single-camera PTV method. As tracer particles, polystyrene spheres with a diameter of 1 or $2 \mu \mathrm{m}$ were used. For the liquids and evaporation conditions analysed, it has been demonstrated experimentally that this type of tracer particles follow faithfully the flow and the effect of Brownian motion or sinking velocity is negligible [10]. For more details about experimental procedures and data processing, we refer to Refs [1012].

\subsection{Ultra pure water}

In the simplest case of evaporation of sessile droplets of ultrapure water (UPW), the capillary flow described by Deegan et al. [4] is the dominant one, pushing the liquid in the bulk toward the contact line. Additionally, a weak but persistent temperature gradient develops across the droplet, since the liquid is cooled down by the evaporation process driving a heat flux from the substrate at larger temperature. The temperature gradient is extremely small, far below 1 $\mathrm{K} / \mathrm{mm}$, but sufficient to drive a thermal Marangoni flow on the surface.

(a)

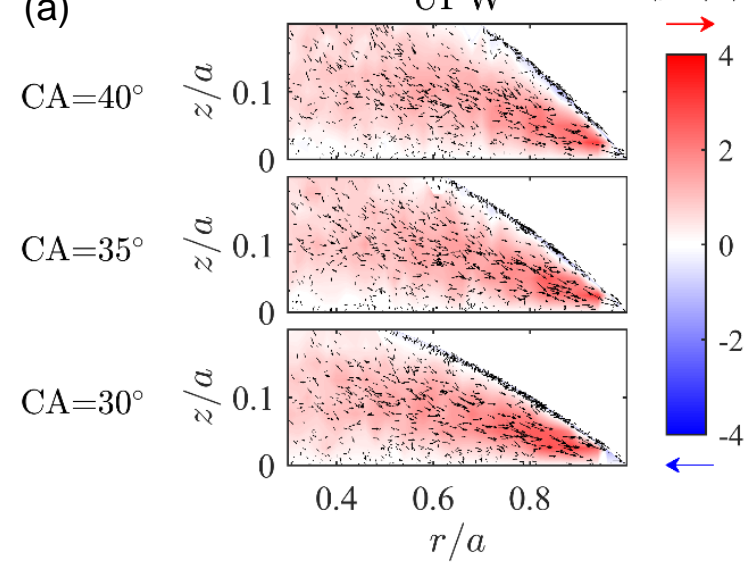

(b)

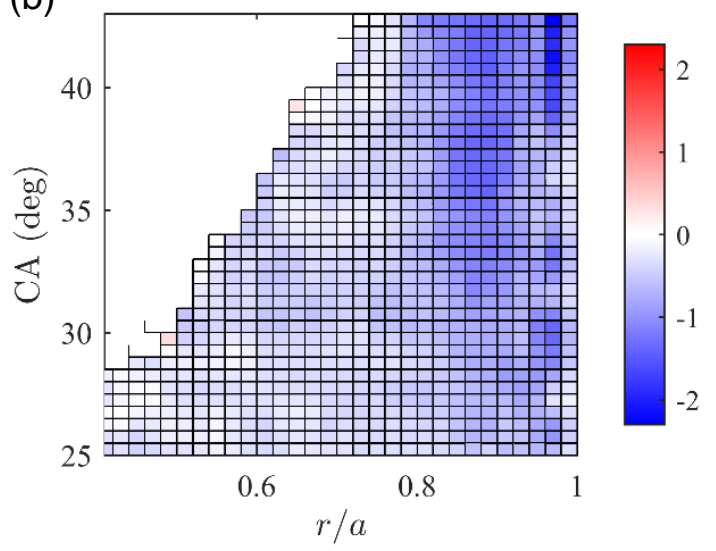

Fig. 4. (a) GDPT measurements of the flow field inside sessile evaporating droplets of UPW, for three different CA, corresponding to different evaporation times. The colormap represents the radial velocity, which is positive when moving toward the contact line. (b) Magnitude of the thermal Marangoni flow on the drop water-air surface as a function of radial position and CA (i.e. time). The largest values are observed close to the contact line and decrease as the drop becomes thinner.

Flow measurements performed with GDPT on a 1-mm-diameter sessile droplet of UPW are shown in Fig. 4(a), for different CAs. Note that since the CA decreases linearly in time, it is used as an equivalent of the remaining evaporation time in Figure 4. The measurements are originally three-dimensional but have been converted to cylindrical coordinates and plotted on the $r z$-plane on account of the axisymmetry of the system. The capillary flow is clearly visible, with the flow velocity increasing as it approaches the contact line, with velocities up to $4 \mu \mathrm{m} / \mathrm{s}$. The thermal Marangoni flow is also present on the surface, directed toward the drop summit. A more detailed picture of the Marangoni flow is given in Fig. 4(b), where the intensity of the superficial flow as a function of radial position and CA is shown. It can be seen as the larger 
magnitude of the Marangoni flow is observed close to the contact line $(r / a=0.9$, with $a$ droplet radius) with values around $2 \mu \mathrm{m} / \mathrm{s}$, and it decreases as the droplet evaporate (i.e. for smaller CA).

\subsection{Mineral water}

The measurements in Fig. 4 are in good qualitative agreement with numerical and analytical simulations, however they show values that are 2-3 orders of magnitude smaller than what predicted by the simulations [10,19-20]. The origin of this disagreement is still an open question and it is often suggested that it is due to the presence of insoluble surfactants. However, this assumption has never been proven experimentally, neither the nature of such insoluble surfactant contaminant identified. One strategy to check the effect of tiny amount of contaminants in the evaporation process, is to look at different types of drinkable water and compare it with the results obtained with UPW.

In Fig.5, the flow velocity fields measured for three types of commercially available mineral waters are reported. The velocity measurements are taken using GDPT and 1- $\mu \mathrm{m}$-diameter polystyrene spheres as tracer particles [10]. The mineral waters, namely $\mathrm{ViO}$, Vittel, and Gerolsteiner, have been chosen with an increasing content of dissolved mineral salts. When the content of mineral salts is low $(\mathrm{ViO})$, the flow patterns and velocity magnitudes are very close to the ones of UPW droplets, suggesting that the superficial flow is still of thermocapillary nature and not affected by the small ionic contamination. For larger content of mineral salts (Vittel and Gerolsteiner), a recirculating flow, moving the fluid in the bottom toward the droplet center, appears. The superficial flow maintains the characteristics of a thermal Marangoni flow (moving from the contact line toward the drop summit), but it starts to slow down, probably because of the onset of the recirculating flow. These results suggest that the presence of tiny amount of contaminants cannot be the only reason for the discrepancy between experiments and simulations, and a more complex thermofluidic scenario must be considered.

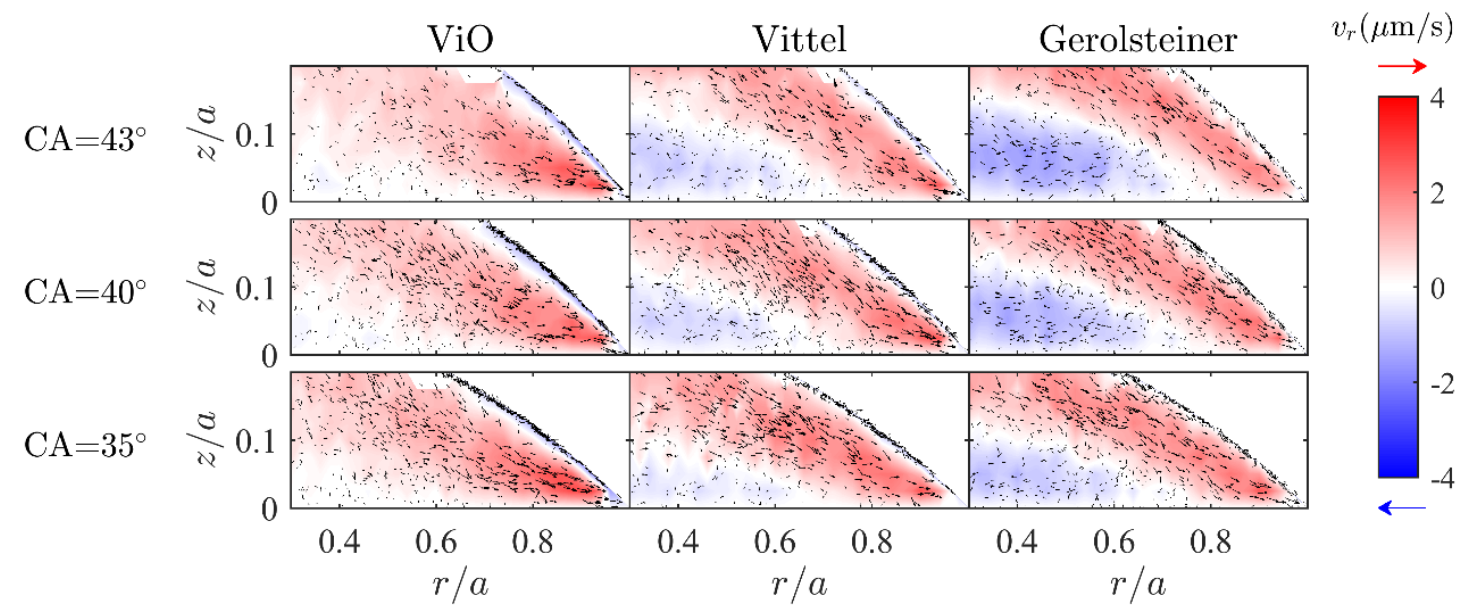

Fig. 5. GDPT measurements of the flow field inside sessile evaporating droplets of three different bottled mineral water with increasing content of dissolved mineral salts: ViO, Vittel, Gerolosteiner. For low content of minerals ( $\mathrm{ViO}$ ), the flow field is comparable to UPW. Increasing the mineral content (Vittel, Gerlosteiner), a stronger and stronger recirculating flow in the bottom of the droplet start to appear, and the thermal Marangoni flow on the surface is slowed down. 


\subsection{Salty solution}

For a larger amount of salt in the water droplets, a reversal of the capillary flow is observed due to the strong solutal Marangoni flow generated by the accumulation of salt at the contact line. This local increase of salt generates a surface tension gradient and consequently a strong flow directed toward the contact line, which consequently drives bulk flow toward the center of the droplet to comply with volume conservation. It has been shown that this flow can also lead to the formation of ring-shaped stains, however the stain morphology is radically different since the particles forming the stain arrive along the liquid-air surface, forming a monolayer of particles along the surface. 3D PTV measurements can be used to reveal this mechanism.

(a)

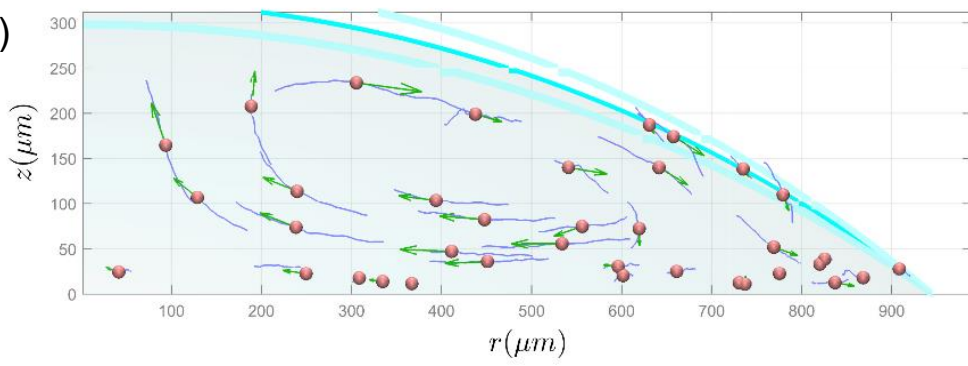

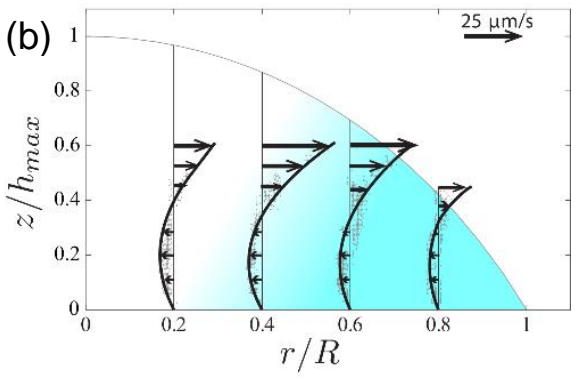

Fig. 6. (a) Trajectory of tracer particle inside a salty water droplet measured with APTV. The droplet has an initial concentration of $100 \mathrm{mM} \mathrm{NaCl}$. A reversed recirculating pattern is observed with superficial flow moving toward the contact line. (b) Corresponding flow velocity profiles derived from the APTV measurements.

Measurements performed with APTV on a salty water droplet are shown in Fig. 6 . The droplet had an initial volume of $0.8 \mu \mathrm{l}$, with initial $\mathrm{CA}$ of $40^{\circ}$ and initial salt $(\mathrm{NaCl})$ concentration of 100 mM. Fig. 6(a) shows some exemplary measured particle trajectories: a reversed circulatory pattern is observed, with particles on the drop surface moving toward the contact line. The corresponding measured flow velocity profiles are shown in Fig. 6(b).

\subsection{Surfactants}

More complex scenarios can be observed in droplets with surfactants due to their complex dynamics in an also dynamic system as an evaporating droplet. Interfacial flows and the continuous droplet surface decrease influences critically the surfactant adsorption/desorption equilibrium at every instant of the evaporation process, giving rise to very complex and changing liquid flow configuration that depends strongly on the surfactant nature. 3D PTV measurements are an indispensable tool to understand the effect of different surfactants at different concentrations. Velocity profiles calculated from APTV measurements on different types of droplets are shown in Fig. 7. In Fig. 7(a), the reference experiment on UPW is shown, with the bulk capillary flow toward the contact line, and the thermal Marangoni flow toward the drop summit. Adding the surfactant Polysorbate 80 (P80) at its critical micelle concentration (CMC) change dramatically this picture. As shown in Fig. 7(b), P80 reduces the surface mobility, creating a rigid droplet surface that drastically reduce the bulk flow as a consequence of increased viscous dissipation. P80 is known to form fairly rigid and stable interfaces when the CMC is reached, which agrees with the results obtained from 3D-PTV measurements. On the contrary, the ionic surfactant Sodium Dodecyl Sulfate (SDS) is known to generate very elastic and mobile interfaces. At 50 times the CMC it promotes strong flows inside the droplet with the establishment of two counter-rotating recirculating flows, as shown in Fig. 7(c). These 
measurements provide a first quantitative picture of these complex phenomena, and provide the basis for the testing and improvement of numerical models and simulations.

(a)

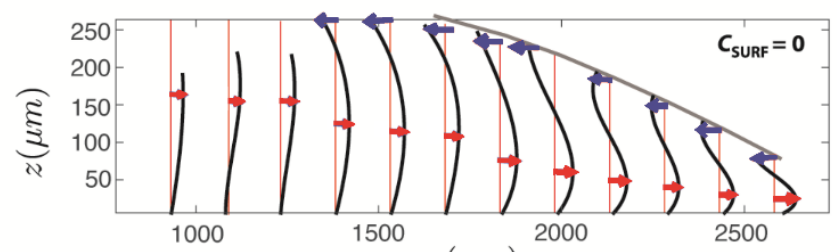

(b)

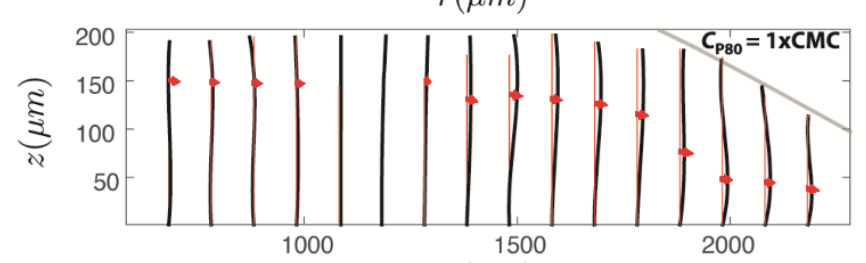

(c)

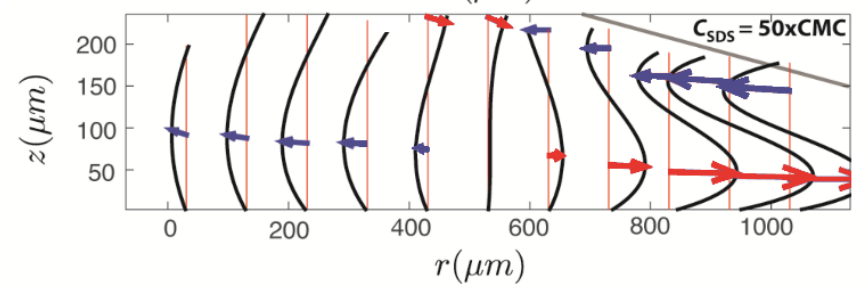

Fig. 7. Experimental velocity profiles obtained from APTV measurements in droplets with different surfactants. (a) Reference case with UPW. (b) Droplet with surfactant P80 at $1 \times C M C$. (c) Droplet with surfactant SDS at $50 \times C M C$.

\section{Conclusions}

We have presented an experimental approach for the quantitative characterization of the 3D flow inside sessile evaporating droplets based on single-camera 3D PTV methods. In particular, we applied two well-established 3D PTV methods based on defocusing: the Astigmatic Particle Tracking Velocimetry (APTV) and the General Defocusing Particle Tracking (GDPT). The effectiveness of this approach is demonstrated by presenting results obtained on different types of sessile droplets, at different experimental conditions. The presented experimental approach can be easily implemented on conventional inverted microscopes, and has the potential to become a standard procedure for these type of measurements.

\section{Acknowledgements}

The authors acknowledge financial support by the Deutsche Forschungsgemeinschaft KA1808/22. MR acknowledges funding from the European Union's Horizon 2020 research and innovation programme under the Marie Sklodowska-Curie grant agreement no. 713683 (COFUNDfellowsDTU). AM acknowledges funding from the European Research Council (ERC-StG-2015 NanoPacks, grant agreement No. 678573).

\section{References}

[1] Zheng, B., Roach, L. S., \& Ismagilov, R. F. (2003). Screening of protein crystallization conditions on a microfluidic chip using nanoliter-size droplets. Journal of the American chemical society, 125(37), 11170-11171.

[2] Sempels, W., De Dier, R., Mizuno, H., Hofkens, J., \& Vermant, J. (2013). Auto-production of biosurfactants reverses the coffee ring effect in a bacterial system. Nature communications, 4 , 1757. 
[3] Shahidzadeh-Bonn, N., Rafal, S., Bonn, D., \& Wegdam, G. (2008). Salt crystallization during evaporation: impact of interfacial properties. Langmuir, 24(16), 8599-8605.

[4] Deegan, R. D., Bakajin, O., Dupont, T. F., Huber, G., Nagel, S. R., \& Witten, T. A. (1997). Capillary flow as the cause of ring stains from dried liquid drops. Nature, 389(6653), 827.

[5] Hu, H., \& Larson, R. G. (2006). Marangoni effect reverses coffee-ring depositions. The Journal of Physical Chemistry B, 110(14), 7090-7094.

[6] Kim, H., Boulogne, F., Um, E., Jacobi, I., Button, E., \& Stone, H. A. (2016). Controlled uniform coating from the interplay of Marangoni flows and surface-adsorbed macromolecules. Physical review letters, 116(12), 124501.

[7] Conn, J. J., Duffy, B. R., Pritchard, D., Wilson, S. K., Halling, P. J., \& Sefiane, K. (2016). Fluiddynamical model for antisurfactants. Physical Review E, 93(4), 043121.

[8] Still, T., Yunker, P. J., \& Yodh, A. G. (2012). Surfactant-induced Marangoni eddies alter the coffeerings of evaporating colloidal drops. Langmuir, 28(11), 4984-4988.

[9] Champougny, L., Scheid, B., Restagno, F., Vermant, J., \& Rio, E. (2015). Surfactant-induced rigidity of interfaces: a unified approach to free and dip-coated films. Soft matter, 11(14), 27582770.

[10] Marin, A., Liepelt, R., Rossi, M., \& Kähler, C. J. (2016). Surfactant-driven flow transitions in evaporating droplets. Soft Matter, 12(5), 1593-1600.

[11] Marin, A., Karpitschka, S., Noguera-Marín, D., Cabrerizo-Vílchez, M. A., Rossi, M., Kähler, C. J., \& Valverde, M. A. R. (2019). Solutal Marangoni flow as the cause of ring stains from drying salty colloidal drops. Physical review fluids, 4(4), 041601.

[12] Rossi, M., Marin, A., \& Kähler, C. J. (2019). Interfacial flows in sessile evaporating droplets of mineral water. Physical Review E, 100(3), 033103.

[13] Cierpka, C., Segura, R., Hain, R., \& Kähler, C. J. (2010). A simple single camera 3C3D velocity measurement technique without errors due to depth of correlation and spatial averaging for microfluidics. Measurement Science and Technology, 21(4), 045401.

[14] Cierpka, C., Rossi, M., Segura, R., \& Kähler, C. J. (2011). On the calibration of astigmatism particle tracking velocimetry for microflows. Measurement Science and Technology, 22(1), 015401.

[15] Rossi, M., \& Kähler, C. J. (2014). Optimization of astigmatic particle tracking velocimeters. Experiments in fluids, 55(9), 1809.

[16] Barnkob, R., Kähler, C. J., \& Rossi, M. (2015). General defocusing particle tracking. Lab on a Chip, 15(17), 3556-3560.

[17] Rossi, M. (2020). Synthetic image generator for defocusing and astigmatic PIV/PTV. Measurement Science and Technology, 31, 017003.

[18] www.defocustracking.com

[19] Hu, H., \& Larson, R. G. (2005). Analysis of the effects of Marangoni stresses on the microflow in an evaporating sessile droplet. Langmuir, 21(9), 3972-3980.

[20] Girard, F., Antoni, M., \& Sefiane, K. (2008). On the effect of Marangoni flow on evaporation rates of heated water drops. Langmuir, 24(17), 9207-9210. 